\title{
artigo
}

Souza, B.F.R.; Buck, E.C.S.; Souza, I.V.B.; Souza, C.R.; Oliveira, R.C.; Morais, C.A.C.;

Cardiopatias congênitas: desafios e perspectivas para o cuidado de enfermagem

\section{Cardiopatias congênitas: desafios e perspectivas para o cuidado de enfermagem}

\author{
Congenital heart diseases: challenges and perspectives for nursing care \\ Enfermedades congénitas del corazón: desafíos y perspectivas de la atención de enfermería
}

\begin{abstract}
RESUMO
Objetivo: Analisar as evidências científicas sobre cuidados de enfemagem à criança com cardiopatia congênita. Método: Trata-se de uma revisão integrativa realizada nas bases de dados MEDLINE, LILACS e BDENF, por meio da BVS, e SciELO no período de agosto a setembro de 2020, a partir da combinação dos descritores selecionados. Foram identificados 39 artigos e, após critérios de inclusão e exclusão, obtiveram-se 9 estudos que compuseram a amostra. Resultados: A partir dos dados emergiram duas categorias temáticas: Atuação da enfermagem no cuidado à criança com cardiopatia congênita e Cuidado à família da criança com cardiopatia congênita. Os estudos destacam a importância dos cuidados sistematizados à criança com cardiopatia congênita, além da crescente necessidade de envolver a família no processo de cuidado.Conclusão: Destaca-se o importante papel da equipe de enfermagem, direcionando a assistência a partir de prioridades estabelecidas o que contribui para a implementação de cuidados integrais e individualizados.
\end{abstract}

DESCRITORES: Cardiopatias congênitas. Assistência de enfermagem. Enfermagem Pediátrica.

\section{ABSTRACT}

Objective: To analyze the scientific evidence on dressing care for children with congenital heart disease. Method: This is an integrative review carried out in the MEDLINE, LILACS and BDENF databases, through the VHL, and SciELO in the period from August to Sep-tember 2020, based on the combination of the selected descriptors. 39 articles were iden-tified and, after inclusion and exclusion criteria, 9 studies were obtained that comprised the sample. Results: From the data, two thematic categories emerged: Nursing perfor-mance in caring for children with congenital heart disease and Care for the family of chil-dren with congenital heart disease. The studies highlight the importance of systematic care for children with congenital heart disease, in addition to the growing need to involve the family in the care process. Conclusion: The important role of the nursing team is high-lighted, directing assistance from established priorities which contributes to the imple-mentation of comprehensive and individualized care.

DESCRIPTORS: Congenital heart disease. Nursing care. Pediatric Nursing.

\section{RESUMEN}

Objetivo: Analizar la evidencia científica sobre el cuidado del vendaje de niños con car-diopatías congénitas. Método: Se trata de una revisión integradora realizada en las bases de datos MEDLINE, LILACS y BDENF, a través de la BVS, y SciELO en el período de agosto a septiembre de 2020, en base a la combinación de los descriptores selecciona-dos. Se identificaron 39 artículos y, luego de criterios de inclusión y exclusión, se obtu-vieron 9 estudios que conformaron la muestra. Resultados: De los datos surgieron dos categorías temáticas: Desempeño de enfermería en el cuidado de niños con cardiopatías congénitas y Atención a la familia de niños con cardiopatías congénitas. Los estudios destacan la importancia de la atención sistemática a los niños con cardiopatías congéni-tas, además de la creciente necesidad de involucrar a la familia en el proceso de cuidado. Conclusión: Se destaca el importante papel del equipo de enfermería, orientando la asis-tencia desde las prioridades establecidas que contribuyen a la implementación de una atención integral e individualizada.

DESCRIPTORES: Cardiopatías congénitas. Cuidado de enfermera. Enfermería pediátrica.

RECEBIDO EM: 21/01/2021 APROVADO EM: 01/02/2021

\section{Beatriz Fernandes Rocha Souza}

Enfermeira graduada pela Faculdade de Enfermagem Nova Esperança.

ORCID: 0000-0001-5855-1946 


\section{Eliane Cristina da Silva Buck}

Enfermeira. Doutoranda em Enfermagem pelo Programa de Pós-graduação em Enfermagem da UFPB.

ORCID: 0000-0002-9230-8760

\section{Ilana Vanina Bezerra de Souza}

Enfermeira. Enfermeira. Mestre pelo Programa de Pós Graduação em Saúde da Família (FACENE).

ORCID: 0000-0002-7833-6415

\section{Carolina Rocha Souza}

Enfermeira graduada pela Faculdade de Enfermagem Nova Esperança.

ORCID: 0000-0002-4717-3190

\section{Regina Célia de Oliveira}

Enfermeira. Pós-Doutorado pela Escola de Enfermagem de Ribeirão Preto.

ORCID: 0000-0002-6559-5872

\section{Camila Abrantes Cordeiro Morais}

Enfermeira. Doutoranda em Enfermagem pelo Programa Associado de Pós-graduação em Enfermagem da UPE-UEPB. ORCID: 0000-0003-3780-9340

\section{INTRODUÇÃO}

A tualmente, os defeitos cardíacos congênitos estão entre as principais causas de morbimortalidade neonatal com prevalência crescente na população. Apesar de todo avanço na assistência, as cardiopatias congênitas (CC) estão associadas ao aumento de perdas fetais, estando presentes em até $85 \%$ dos óbitos em natimortos, recém-nascidos e lactentes. ${ }^{1-2}$

A incidência das CC é de 8 a 10 por mil nascidos vivos, ou 1 em cada 100 nascimentos, variando entre $0,8 \%$ nos países mais desenvolvidos a $1,2 \%$ nos países subdesenvolvidos. No Brasil, cerca de 28.900 crianças nascem com CC por ano (1\% do total de nascimentos), das quais cerca de 80\% (23.800) necessitam de cirurgia cardíaca, sendo metade destas no primeiro ano de vida. ${ }^{3-4}$

Os distúrbios cardíacos congênitos estão relacionados, frequentemente, à ocorrência de insuficiência cardíaca, ao comprometimento do sistema pulmonar e à hipoxemia. A assistência de uma equipe multiprofissional é essencial diante do processo de cuidar da criança com CC, com destaque para o enfermeiro, que realiza o cuidado integral a partir de um planejamento da assistência, considerando que tais condições clínicas necessitam de intervenções individualizadas que incluem a manutenção e a monitorização das fun- ções cardíaca e pulmonar, do acúmulo de líquidos e eletrólitos e de medidas relacionadas à prevenção de infecção. ${ }^{5-6}$

Diante das complicações associadas às $\mathrm{CC}$ e das consequências decorrentes das frequentes hospitalizações, é importante destacar que a doença apresenta repercussão não apenas na vida da recém-nascido, mas também na família, sendo comum a presença de ansiedade, sentimentos de impotência, culpa e medo, o que torna essencial o apoio dos profissionais de saúde, fornecendo orientações acerca da saúde e tratamento?

Além dos cuidados direcionados a criança e a necessidade de incluir a família no processo, a equipe de enfermagem é considerada essencial na investigação acerca do diagnóstico de CC, pois a avaliação clínica destes profissionais permite a identificação precoce de manifestações clínicas consideradas importantes no período neonatal, tais como: presença de sopro cardíaco, taquipneia progressiva, cansaço ás mamadas, cianose, hipotensão e arritmias cardíacas. ${ }^{8}$

De forma geral, após a confirmação do diagnóstico da $\mathrm{CC}$, há a indicação para a correção cirúrgica, sendo esta dependente de vários fatores como: idade, peso, condições nutricionais, tipo da cardiopatia e intervenção cirúrgica propriamente dita. Assim, compreendendo a complexidade das atividades assistenciais prestadas ao pa- ciente cardiopata, a utilização do Processo de Enfermagem (PE) como instrumentalização da Sistematização da Assistência de Enfermagem (SAE) é de fundamental importância subsidiar o diagnóstico de enfermagem, planejamento e implementação das intervenções de acordo com a necessidade do indivíduo e família. ${ }^{9}$

Nessa perspectiva, o estudo é relevante para ampliar o conhecimento de profissionais da saúde acerca da temática, contribuindo para a qualidade da assistência de enfermagem à criança com CC ao favorecer a elaboração de estratégias e intervenções que possam atender às reais necessidades dessa população. Sendo assim, este estudo tem como objetivo analisar as evidências científicas sobre cuidados de enfermagem à criança com cardiopatia congênita.

\section{MÉTODOS}

Trata-se de uma Revisão Integrativa da Literatura, em que foram percorridas as seguintes etapas: identificação de problema, com a definição da questão de pesquisa; estabelecimento de critérios para inclusão e/ou exclusão de estudos para a busca de literatura científica; definição das informações a serem extraídas dos estudos; avaliação dos estudos; interpretação dos resultados e apresentação da revisão/síntese do conhecimento ${ }^{10}$. Atendendo ao ob- 


\section{artigo}

Souza, B.F.R.; Buck, E.C.S.; Souza, I.V.B.; Souza, C.R.; Oliveira, R.C.; Morais, C.A.C.;

Cardiopatias congênitas: desafios e perspectivas para o cuidado de enfermagem

jetivo anteriormente descrito, foi definida a seguinte questão de pesquisa: "Quais as evidências científicas sobre os cuidados de enfermagem a criança com cardiopatia congênita?".

A busca foi realizada em três bases de dados: Medical Literature Analysis and Retrieval System Online (MEDLINE), Literatura Latino-Americana do Caribe em Ciências da Saúde (LILACS) e Bases de Dados de Enfermagem (BDENF) por meio do Portal da Biblioteca Virtual em Saúde (BVS). Para melhor selecionar os arquivos, também foi utilizado o banco de periódicos Scientific Electronic Library Online (SciELO).

A busca foi operacionalizada a partir da utilização de termos identificados no vocabulário na base dos Descritores em Ciências da Saúde (DeCS) e no Medical Subject Headings (MeSH). Assim, foi utilizada uma combinação de descritores: "Cardiopatias congênitas"; "Assistência de Enfermagem";
"Enfermagem pediátrica", separados pelo operador booleano "AND".

A coleta de dados ocorreu no período de agosto a setembro de 2020, sendo realizada por dois pesquisadores, de forma independente. Foram definidos como critérios de inclusão: publicações na modalidade de artigo, texto completo, publicados no período de 2010 a 2020, nos idiomas português, espanhol e inglês. Refere-se que foram excluídas publicações como: teses, dissertações, monografias, trabalhos de conclusão de curso, revisão de literatura, manuais, notas prévias e estudos que não abordavam a temática proposta.

Para a seleção dos estudos seguimos as recomendações do método Preferred Reporting Items for Systematic reviews and Meta-Analyses - PRISMA ${ }^{11}$ (Figura 1). Assim, os artigos foram inicialmente selecionados pelo título e resumo e, posteriormente, lidos na íntegra, incluindo-se os que continham informações relevan-
Figura 1: Fluxograma do processo de identificação, seleção e inclusão dos artigos, adaptado do PRISMA.

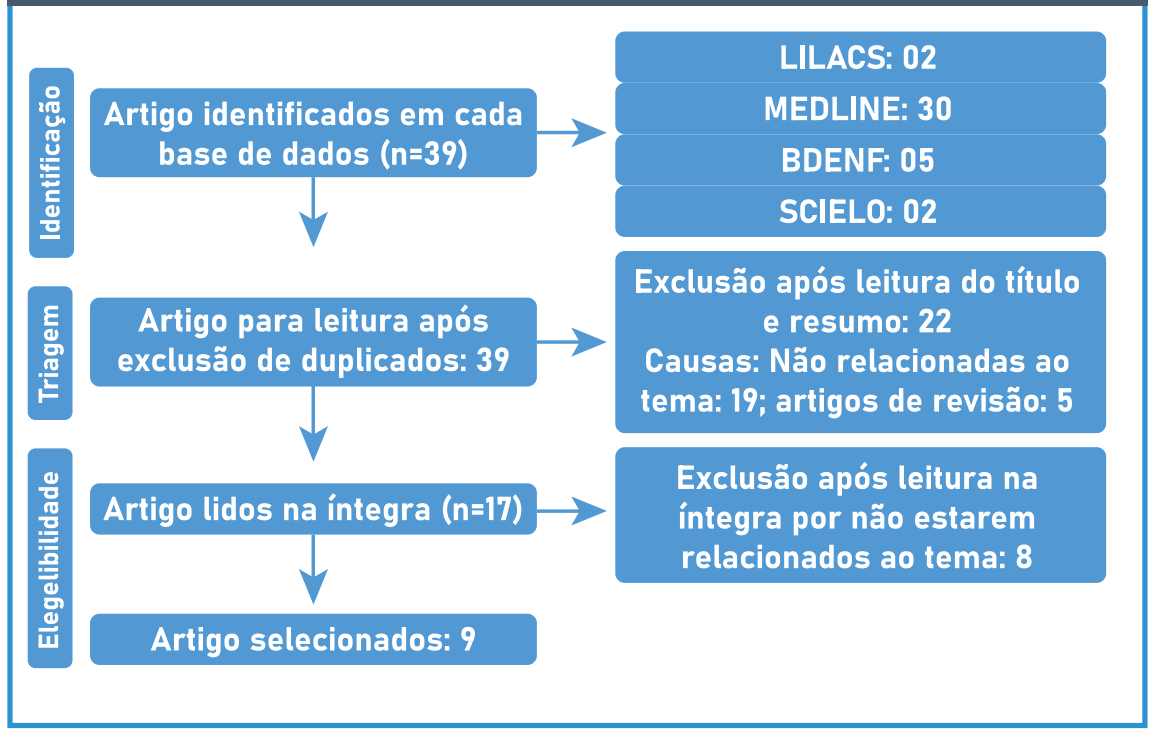

tes acerca dos cuidados de enfermagem a criança com cardiopatia congênita.

Os resultados foram dispostos por meio de quadros e agrupados em categorias temáticas. A análise de artigos foi realizada de forma descritiva, com a síntese das evidências de cada publicação.

\section{RESULTADOS}

Inicialmente, foi identificado um total de 39 publicações. Após as etapas de identificação, triagem e elegibilidade, a amostra foi composta por 9 artigos. A Figura 1 apresenta um fluxograma do processo de busca e seleção dos artigos por base de dados.

Para facilitar a análise dos dados, foi utilizado instrumento adaptado da literatura pelos autores, que contempla características de identificação do artigo tais como: título, autores, ano, base de dados, descrição metodológica (tipo de estudo e abordagem) e o objetivo.

Em relação às bases de dados onde os artigos estavam indexados, a MEDLINE foi a que teve mais publicações, seis (60\%). Pôde-se constatar que oito (80\%) artigos foram publicados em periódicos internacionais e dois $(20 \%)$ em periódicos nacionais. O periódico onde houve o maior número de publicações foi a Revista American Journal of Critical Care, com três estudos (30\%).

Em relação às bases de dados onde os artigos estavam indexados, a MEDLINE foi a que teve mais publicações, seis (60\%). Pôde-se constatar que oito (80\%) artigos foram publicados em periódicos internacionais e dois (20\%) em periódicos nacionais. O periódico onde houve o maior número de publicações foi a Revista American Journal of Critical Care, com três estudos (30\%), conforme observado no Quadro 1.

\section{Quadro 1: Distribuição dos artigos selecionados para a revisão integrativa. João Pessoa (PB), Brasil, 2020.}

\begin{tabular}{|c|c|c|}
\hline ANO & AUTOR/TíTULO/PERÍODICO & BASE DE DADOS \\
\hline 2019 & $\begin{array}{c}\text { Uhm JY, Choi MY. Mothers' needs regarding partnerships with nurses during care of infants with congenital } \\
\text { heart defects in a paediatric cardiac intensive care unit. Intensive Care Nursing. }\end{array}$ & MEDLINE \\
\hline 2017 & $\begin{array}{r}\text { Bruce E, Sundin K. Pediatric Nurses' Perception of Support for Families With Children With Congenital Heart } \\
\text { Defects. Clinical Nursing Research. }{ }^{13}\end{array}$ & MEDLINE \\
\hline
\end{tabular}




\begin{tabular}{|c|c|c|}
\hline 2017 & $\begin{array}{l}\text { Lisanti AJ et al. Maternal Stress and Anxiety in the Pediatric Cardiac Intensive Care Unit. American Journal of } \\
\text { Critical Care. }{ }^{14}\end{array}$ & MEDLINE \\
\hline 2016 & $\begin{array}{l}\text { Connor JA et al. Measurement of Quality Pratice in Congenital Cardiac Care. American Journal of Critical } \\
\text { Care. }{ }^{15}\end{array}$ & MEDLINE \\
\hline 2015 & $\begin{array}{l}\text { Silva VG et al. Diagnósticos de Enfermagem em crianças com cardiopatias congênitas: mapeamento cruzado. } \\
\text { Acta Paulista de Enfermagem. }{ }^{16}\end{array}$ & SCIELO \\
\hline 2013 & $\begin{array}{l}\text { Hickey PA. The Effect of Critical Care Nursing and Organizational Characteristics on Pediatric Cardiac Surgery } \\
\text { Mortality in the United States. The Journal of Nursing Administration. }{ }^{17}\end{array}$ & MEDLINE \\
\hline 2012 & $\begin{array}{l}\text { Melo HC et al. O Ser- Enfermeiro em face do cuidado à criança no pós operatório imediato de cirurgia cardía- } \\
\text { ca. Esc. Anna Nery Rev. Enferm. }{ }^{18}\end{array}$ & LILACS \\
\hline 2012 & $\begin{array}{c}\text { Hickey PA et al. Pediatric Cardiovascular Critical Care in the Unidt States: Nursing and Organizational Charac- } \\
\text { teristics. American Journal of Critical Care. }{ }^{19}\end{array}$ & MEDLINE \\
\hline 2011 & $\begin{array}{c}\text { Benítez ERD. Lactante menor con alteración de las necesidades básicas secundario a cardiopatía congénita. } \\
\text { Revista Mexicana de Enfermería Cardiológica. }{ }^{20}\end{array}$ & BDENF \\
\hline
\end{tabular}

Dos nove artigos selecionados, notou-se que a média de publicação no recorte temporal aplicado foi de um a dois artigos por ano, porém, nos anos de 2012 e 2017, foram publicados dois artigos (20\%), seguidos de uma publicação nos demais anos. Ressalta-se que, nos anos de 2010 e 2014, não foi encontrada nenhu- ma publicação que atendesse aos critérios deste estudo. No Quadro 2, apresentamos a síntese dos estudos incluídos nesta revisão, quanto ao método utilizado nas pesquisas e o objetivo de cada publicação. Houve predomínio de estudos descritivos $(66,6 \%)$, sendo a abordagem qualitativa a mais prevalente $(55,5 \%)$.

\section{DISCUSSÃO}

Os resultados foram agrupados em duas categorias temáticas considerando os achados mais relevantes: Atuação da enfermagem no cuidado à criança com cardiopatia congênita; Cuidado à família da criança com cardiopatia congênita.

Quadro 2: Distribuição dos estudos acerca dos cuidados de enfermagem à criança com cardiopatia congênita, segundo características dos artigos. João Pessoa (PB), Brasil, 2020.

\begin{tabular}{|c|c|c|}
\hline $\mathbf{N}^{\circ}$ & TIPO DE ESTUDO E ABORDAGEM & OBJETIVO \\
\hline E1 & Estudo descritivo de abordagem quantitativa. & $\begin{array}{l}\text { Investigar as necessidades das mães na formação de parcerias com enfermeiras, } \\
\text { com base na recuperação pós operatória em UTI cardíaca pediátrica. }\end{array}$ \\
\hline E2 & Estudo descritivo de abordagem qualitativa. & $\begin{array}{l}\text { Conhecer as percepções dos enfermeiros pediátricos (PNs) de apoio às familias } \\
\text { com uma criança com defeito cardíaco congênito. }\end{array}$ \\
\hline E3 & Estudo descritivo de abordagem quantitativa. & $\begin{array}{l}\text { Explorar os estressores e a resposta ao estresse de mães cujos bebês com car- } \\
\text { diopatia congênita estavam sendo cuidados em UTI cardíaca pediátrica. }\end{array}$ \\
\hline E4 & Estudo descritivo de abordagem qualitativa. & $\begin{array}{l}\text { Avaliar o estado atual de medição do qualidade da enfermagem cardiovascular } \\
\text { pediátrica em hospitais infantis independentes nos Estados Unidos. }\end{array}$ \\
\hline E5 & $\begin{array}{l}\text { Estudo observacional transversal, de } \\
\text { abordagem quantitativa. }\end{array}$ & $\begin{array}{l}\text { Identificar Diagnósticos de Enfermagem da NANDA a partir dos termos encon- } \\
\text { trados nos registros de Enfermagem de crianças com cardiopatias congênitas } \\
\text { hospitalizadas e verificar associação entre estes termos e os Diagnósticos de } \\
\text { Enfermagem mapeados. }\end{array}$ \\
\hline E6 & Estudo descritivo de abordagem qualitativa. & $\begin{array}{l}\text { Este estudo explorou pediatria crítica cuidados de enfermagem e fatores or- } \\
\text { ganizacionais que impactam mortalidade hospitalar para pacientes de cirurgia } \\
\text { cardíaca em hospitais infantis nos Estados Unidos. }\end{array}$ \\
\hline E7 & Estudo descritivo de abordagem qualitativa. & $\begin{array}{c}\text { Compreender o cuidado à criança durante o pós-operatório imediato de cirurgia } \\
\text { cardíaca a partir da percepção do ser-enfermeiro. }\end{array}$ \\
\hline E8 & Estudo descritivo de abordagem quantitativa & $\begin{array}{l}\text { Descrever e compreender as características de enfermagem e organizacionais } \\
\text { dos cuidados cardiovasculares em hospitais infantis. }\end{array}$ \\
\hline E9 & Estudo retrospectivo de aborgaem qualitativa. & $\begin{array}{l}\text { Identificar as necessidades básicas de um bebê com doença cardíaca congênita e } \\
\text { desenvolver um processo de enfermagem com a abordagem de Virginia Henderson. }\end{array}$ \\
\hline
\end{tabular}


Atuação da enfermagem no cuidado à criança com cardiopatia congênita

Com o objetivo de correlacionar padronização de diagnósticos, intervenções e resultados de enfermagem frente aos cuidados sistematizados à criança com cardiopatia congênita, estudo ${ }^{16}$ destacou os seguintes diagnósticos de enfermagem: diminuição do débito cardíaco, risco de infecção, padrão respiratório ineficaz, intolerância a atividade, troca de gases prejudicada. Também ressaltou a importância do reconhecimento precoce das complicações, além de garantir monitorização de pressão arterial, padrão respiratório, oximetria de pulso, frequência respiratória, manutenção do suporte ventilatório mecânico, entre outros. ${ }^{16}$

Tendo em consideração a complexidade dos defeitos cardiacos congênitos, os pacientes são mais propícios a infecções, o que pode proporcionar um aumento no tempo de internação e maior taxa de mortalidade. Vários elementos podem proporcionar o desenvolvimento das infecções hospitalares nas crianças, como a demorada maturação do sistema imunológico, a partição de objetos entre pacientes pediátricos, a desnutrição, o aparecimento de anomalias congênitas, a medicação (principalmente corticosteroides) e as doenças hemato-oncológicas. ${ }^{17}$

A área de Enfermagem em cardiologia pediátrica exige uma equipe altamente qualificada, com conhecimento e experiência necessários para o manejo de possíveis complicações nessa população, necessitando de medidas que demonstrem o impacto dos cuidados prestados aos pacientes e familiares. Assim, foi criado o Consórcio de Cuidados Cardíacos Congênitos- Medição da Prática de Enfermagem (C4-MNP), no qual foram selecionados líderes de enfermagem com experiência clínica, administrativa e de pesquisa de programas cardiovasculares pediátricos nos Estados Unidos, com o objetivo de identificar ações ou medidas de cuidados de enfermagem em lugares complexos de cuidados cardiovasculares pediátricos. ${ }^{15,17}$

Um estudo realizado com o objetivo
Vários elementos podem proporcionar o desenvolvimento das infecções hospitalares nas crianças, como a demorada maturação do sistema imunológico, a partição de objetos entre pacientes

pediátricos, a

desnutrição, o

aparecimento

de anomalias

congênitas,

a medicação

(principalmente

corticosteroides) e

as doenças hemato-

oncológicas. de compreender o cuidado à criança durante o pós-operatório imediato de cirurgia cardíaca a partir da percepção do ser-enfermeiro evidenciou que o cuidado à criança é concebido através da relação entre tecnologias duras e subjetividade, mas, quando o foco da assistência é voltado para a doença e para procedimentos envolvidos no tratamento, o cuidado perde a sua totalidade, pois as dimensões humanas que o envolvem não adquirem seu devido destaque. ${ }^{18}$

\section{Cuidado à família da criança com cardiopatia congênita}

Com relação ao apoio de familiares de crianças com CC, autores reforçam a importância do enfermeiro no suporte em permitir o envolvimento dos pais no processo do cuidado dessa criança, tornando-os ativos nesse âmbito. Nesse sentido, o enfermeiro deve informar os pais sobre o cuidado da criança, criar um bom relacionamento com a família e construir a confiança de todas as partes envolvidas. Além disso, a equipe de enfermagem deve proporcionar segurança e autoestima à familia durante todo atendimento da criança; assim, torna-se uma relação de confiança com a equipe e com outros familiares, facilitando o cuidado e exaurindo a tensão. ${ }^{13-14}$

Além disso, destaca-se o enfermeiro na observação desses fatores, pois isso gera um impacto na vida adulta, onde a equipe de enfermagem e os pais devem implementar intervenções que possibilitem o desenvolvimento psicomotor. Os resultados de um estudo ${ }^{20}$ enfatizam que as crianças necessitam de contato físico e estimulação para sua recuperação ou cooperação no tratamento. Assim, a equipe de enfermagem e os pais devem ser parceiros: os pais são fundamentais para dar o apoio e a autorização para a prática da assistência de enfermagem.

É estabelecido que a parceria entre pais e enfermeiras é um relacionamento baseado na cooperação uns com os outros para fornecer saúde infantil ideal e cuidado. As informações, quando compartilhadas, proporcionam que os cuidados sejam exe- 
cutados de uma forma facilitada. Dentro de unidades de terapia intensiva neonatal e pediátrica, os pais necessitam de esclarecimentos precisos, oportunos e compreensíveis, especialmente as mães, que eventualmente tendem a ficar com seus filhos por mais tempo e participam da execução dos cuidados prestados à criança. ${ }^{12}$

\section{CONCLUSÃO}

Diante do objetivo proposto, evidencia-se a importância da assistência de enfermagem à criança com cardiopatia congênita quanto à identificação precoce de sinais e sintomas, além da realização de ações voltadas à prevenção e ao tratamento de complicações.

O estudo também aponta à necessida-
O estudo também aponta à necessidade crescente de envolver a família no cuidado à criança com $\mathrm{CC}$... de crescente de envolver a família no cuidado à criança com CC, tendo em vista que os profissionais de saúde devem atuar junto aos cuidadores familiares desenvolvendo estratégias para implementação de cuidados, promoção e recuperação da saúde. $\mathrm{O}$ reduzido número de publicações na amostra constitui uma limitação, sendo necessário o desenvolvimento de novas pesquisas que auxiliem na melhoria da qualidade da assistência à criança com CC, considerando a relevância deste problema na infância.

\section{REFERÊNCIAS}

1. Medeiros AL, Freitas TB, Araújo JSS, Mattos SS. Oximetria de pulso em triagem de Cardiopatias Congênitas: Conhecimento e atuação do enfermeiro. Cogitare Enferm. 2015;20(3):605-11.

2. Lopes SAVA, Guimarães ICB, Costa SFO, Acosta AX, Sandes KA, Mendes MC. Mortalidade para Cardiopatias Congênitas e Fatores de Risco Associados em Recém-Nascidos. Um Estudo de Coorte. Arq Bras Cardiol. 2018;111(5):666-73.

3. Brasil. Ministério da Saúde. Síntese de evidências para políticas de saúde: diagnóstico precoce de cardiopatias congênitas. Brasília: Ministério da Saúde, 2017.

4. Soares AM. Mortalidade para Cardiopatias Congênitas e Fatores de Risco Associados em Recém-Nascidos. Um Estudo de Coorte. Arq Bras Cardiol. 2018;111(5):674-75.

5. Almeida FA, Margoni AP, Santos MA. Compreendendo a experiência de cuidar da criança com cardiopatia congênita. Invest Qualit Saúde. 2016;(2):1663-72.

6. Magalhães SS, Queiroz MVO, Chavez EMC. Cuidados da enfermagem neonatal ao bebê com cardiopatia congênita: revisão integrativa. Online braz j nurs. 2016;15(4):724-34.

7. Chaves RRG, Silva CFM, Motta E, Ribeiro EDLM, Andrade YNL. Sistematização da Assistência de Enfermagem: visão geral dos enfermeiros. Rev Enferm UFPE. 2016;10(4):1280-5.

8. Lima TG, Silva MA, Siqueira SMC. Diagnósticos e cuidados de enfermagem ao neonato com cardiopatia congênita. Rev Soc Cardiol Estado de São Paulo - Supl - 2018;28(1):101-9.

9. Cabral JVB, Chaves JSC. Cuidado de enfermagem no pós-operatório de cirurgia cardíaca pediátrica: revisão integrativa. Rev Enferm Contemp. 2020;9(1):118-126.

10. Souza MT, Silva MD, Carvalho R. Revisão Integrativa: o que é e como fazer. Einstein. 2010;8(1):102-06.

11. Moher D, Liberati A, Tetzlaff J, Altman DG; PRISMA Group. Preferred reporting items for systematic reviews and meta-anal- yses: the PRISMA statement. Int J Surg. 2010;8(5):336-41.

12. Uhm JY, Choi MY. Mothers' needs regarding partnerships with nurses during care of infants with congenital heart defects in a pediatric cardiac intensive care unit. Intensive Care Nursing. 2019;54:79-87.

13. Bruce E, Sundin K. Pediatric Nurses' Perception of Support for Families With Chil-dren With Congenital Heart Defects. Clin Nurs Res. 2018; 27(8):950-966.

14. Lisanti AJ, Allen LR, Kelly L, Medoff-Copper B. Maternal Stress and Anxiety in the Pediatric Cardiac Intensive Care Unit. American Journal of Critical Care. 2017;26(2):118-23.

15. Connor JA, Mott S, Green A, Larson C, Hickey P. Measurement of Quality Pratice in Congenital Cardiac Care. American Journal of Critical Care. 2016;25(2):128-35.

16. Silva VG, Pereira JMV, Figueiredo LS, Guimarães TCF, Cavalcanti ACD. Diagnósticos de Enfermagem em crianças com cardiopatias congênitas: mapeamento cruzado. Acta Paul Enferm. 2015;28(6):524-30.

17. Hickey PA, Gauvreu K, Tong E, Schiffer NP, Connor JA. Pediatric Cardiovascular Crit-ical Care in the Unidt States: Nursing and Organizational Characteristics. American Journal of Critical Care. 2012;21(4):242-50.

18. Melo HC, Araújo SEG, Santos VEFA, Veríssimo AVR, Alves ERP, Souza MHN. O Ser-Enfermeiro em face do cuidado à criança no pós operatório imediato de cirurgia cardíaca. Esc. Anna Nery Rev. Enferm. 2012;16(3):473-79.

19. Hickey PA, Gauvreu K, Tong E, Schiffer NP, Connor JA. Pediatric Cardiovascular Crit-ical Care in the Unidt States: Nursing and Organizational Characteristics. American Jour-nal of Critical Care. 2012;21(4):242-50.

20. Benítez, ERD. Lactante menor con alteración de las necesidades básicas secundario a cardiopatía congénita. Revista Mexicana de Enfermería Cardiológica. 2011;19(2):74-79. 